\title{
Moyamoya disease with early-onset achalasia
}

INSERM

\section{Source}

INSERM. (1999). Orphanet: an online rare disease and orphan drug data base.

Moyamoya disease with early-onset achalasia. ORPHA:401945

Moyamoya disease with early-onset achalasia is an exceedingly rare autosomal recessive neurological disorder reported only in a few families so far. It is characterized by the association of early onset achalasia (manifesting in infancy) with severe intracranial angiopathy that is consistent with moyamoya angiopathy in most cases (moyamoya disease; see this term). Other variable associated manifestations include hypertension, Raynaud phenomenon, and livedo reticularis. 